\title{
Decision Support Pipelines - Durchgängige Datenverarbeitungsinfrastrukturen für die Entscheidungen von morgen
}

Anne Meyer, Stefan Zander, Rico Knapper und Thomas Setzer

\subsection{Einleitung}

Unterschiedliche, sich schnell entwickelnde Technologien aus dem Bereich Industrie 4.0 erlauben die immer detailliertere Aufnahme von Daten, die den aktuellen Zustand von Geschäfts-, Produktions- oder Logistikprozessen beschreiben. Die resultierende Flut an Daten allein bietet einem Unternehmen jedoch unmittelbar noch keine Vorteile. ${ }^{1}$ Nur wenn die Daten tatsächlich zur Basis für bessere Unternehmensentscheidungen werden - zum Beispiel durch das Gewinnen problemrelevanter Erkenntnisse für menschliche Entscheider oder durch die Unterstützung durch (teil-)automatisierte Planungs- und Steuerungsverfahren wird der Einsatz von Industrie 4.0 Technologien wirksam (vgl. Schreiner 2013; Laney 2015).

Der Prozess der Datentransformation ist damit offensichtlich erfolgskritisch für alle Bemühungen im Bereich Industrie 4.0 (vgl. European Commission 2013 und http://www. fofdationproject.eu/). In sich dynamisch ändernden, oft komplexen Geschäftsumfeldern

${ }^{1}$ Lt. einer Untersuchung des Marktforschungsunternehmens Pierre Audoin Consultants (PAC) sehen $55 \%$ der Befragten Unternehmen die permanent steigende Datenflut als eine der größten Herausforderungen für Industrie 4.0 (Prud'hommeaux und Seaborne PAC 2013).

\footnotetext{
A. Meyer $(\square)$

FZI Forschungszentrum Informatik, Haid-und-Neu-Str. 10-14, 76131 Karlsruhe, Deutschland e-mail: meyer@fzi.de
}

S. Zander

Fachbereich Informatik, Hochschule Darmstadtc, Schöfferstraße 8B, 64295 Darmstadt, Deutschland e-mail: stefan.zander@h-da.de

R. Knapper · T. Setzer 
weist schon die isolierte Betrachtung einzelner Aktivitäten und Teilprozesse beispielsweise zur Gewinnung und Integration von Daten aus unterschiedlichsten.

Quellen, zur Analyse dieser Daten, zum Treffen von (guten) Entscheidungen sowie zur Kommunikation der Entscheidung an den Ort der Umsetzung eine sehr hohe Komplexität auf.

Die Erfahrungen des FZI Forschungszentrum Informatik aus einer Vielzahl an Industrieund Forschungsprojekten im Kontext von Industrie 4.0 und Big/Smart Data zeigen jedoch, dass das enorme Potenzial vorhandener Daten nur sinnvoll genutzt werden kann, wenn die Datenverarbeitung als zusammengehöriger, abgestimmter Prozess - von der Datengewinnung bis zur Entscheidung - verstanden und modelliert wird. Die besondere Herausforderung einer solchen durchgängigen, ganzheitlichen Prozessbetrachtung liegt in der Orchestration von Techniken verschiedener Disziplinen und Forschungsrichtungen wie Wissensmanagement und -repräsentation, skalierbare Infrastrukturen für heterogene, multimodale Datenströme, Data Analytics, statistisches Lernen und Operations Research (vgl. Beste 2014).

Aktuell verfügbare Lösungsangebote auf dem Markt sowie Forschungsprojekte in diesem Bereich adressieren jedoch oft sehr konkrete Anwendungen oder nur einzelne der oben genannten Schritte, die oftmals nicht oder nur bedingt auf andere Aufgaben übertragbar sind. Dieser Beitrag verfolgt daher das Ziel, technologische und methodologische Ansatzpunkte für die Entwicklung einer intelligenten,auf die Anforderungen zukünftiger Industrie 4.0 Fertigungs- und Logistikprozesse ausgerichteten "Echtzeit InformationsWertschöpfungskette" zu liefern. Die dargestellte Vision umfasst neue Methoden und Techniken zur gewinnbringenden Gestaltung durchgehend informationsgetriebener, unternehmerischer Entscheidungen. Damit sollen insbesondere

(i) schnelle Reaktionen auf Veränderungen und Anomalien möglich sein,

(ii) mehr Daten aus heterogenen Quellen in Echtzeit verarbeitet werden können,

(iii) Zusammenhange auch in größeren Datenmengen zuverlässiger und zielgerichteter erkannt werden und

(iv) vorhandene Informationen mit mathematischen und ökonomischen Mechanismen systematisch kombiniert und zur besseren Planung und Steuerung nutzbar gemacht werden.

Hierfür ist die Schaffung technischer Werkzeuge notwendig, die diese Methoden und Techniken als Gesamtkette umsetzen und vom Business Anwender bedient werden können. ${ }^{2}$ Daher sind einfach bedienbare Konfiguratoren und Informationssysteme nötig, auf deren Basis die komplexe Modellbildung bestmöglich unterstützt wird und im Hintergrund abläuft, um den Entscheider von automatisierbaren Schritten zu entlasten und den Fokus gezielt auf notwendige Entscheidungsalternativen zu lenken.

Auf Basis existierender Arbeiten und unseren Erfahrungen aus konkreten Projekten im Kontext Industrie 4.0 charakterisieren wir in diesem Beitrag die typischen Bestandteile

\footnotetext{
${ }^{2}$ Vgl. den Beitrag „Der Mensch in Interaktion mit autonomen Planungs- und Steuerungssystemen für Cyber-Physische Produktionssysteme“ von Susanne Vernim, Christiane Dollinger, Andreas Hees und Gunther Reinhart in diesem Buch.
} 
solcher „Entscheidungssysteme“. Daneben werden die Herausforderungen - sowohl auf den einzelnen Stufen als auch übergreifend - beschrieben und ein Konzept skizziert, um die diversen Anforderungen strukturiert adressieren zu können. Ein besonderer Fokus liegt darauf, die Wechselwirkungen zwischen den Stufen zu identifizieren (Abschn. 15.4). Für konkrete Anwendungsfälle betrachten wir das Potenzial einer Decision Support Pipeline (Abschn. 15.3) und schließen den Beitrag mit einer kurzen Zusammenfassung (Abschn. 15.4).

\subsection{Durchgängige Datenverarbeitungskette}

Basierend auf Auswertungen vergangener und aktueller Projekte aus unterschiedlichen Themenbereichen im Kontext Industrie 4.0 diskutieren wir in diesem Abschnitt Eigenschaften und Anforderungen an eine durchgängige, datengetriebenen Verarbeitungskette sowie deren Manifestation in einer Decision Support Pipeline. Hierbei setzen wir auf ein breites Themenspektrum auf und adressieren Herausforderungen aus den Bereichen Steuerung und Planung fertigungsnaher Logistiknetzwerke (ProveIT, ${ }^{3} \mathrm{RAN}^{4}$ ), dem Störungsmanagement im Produktionsumfeld $\left(\right.$ BigPro $\left.^{5}\right)$, der optimierten Steuerung von fördertechnischen Anlagen (Forschungskooperation Dillinger Hütte ${ }^{6}$ ), der intelligenten Wartungsüberwachung und -steuerung von Industrieanlagen (ProaSense, ${ }^{7}$ BigPro), der Planungsunterstützung öffentlicher Einsatze bei Großschadenlagen (BigGIS ${ }^{8}$ ), sowie der intelligenten Konfiguration von Robotikanlagen und deren Anpassung an individuelle Produktionsgegebenheiten $\left(\operatorname{ReApp}^{9}\right)$.

\subsubsection{Eigenschaften und Anforderungen}

Der Datenverarbeitungsprozess lässt sich auf grobgranularer Betrachtungsebene in fünf aufeinanderfolgende Prozessschritte unterteilen (siehe Abb. 15.1). Zu Beginn steht die (I) Gewinnung und (II) Integration großer Mengen oftmals unstrukturierter und unzuverlässiger Daten aus heterogenen Datenquellen im Vordergrund. Als Quellen stehen typischerweise lokale Systeme genauso wie ubiquitäre Sensoren oder Wissensbasen mit Expertenwissen zur Verfügung. Basierend auf den aus den ersten beiden Schritten resultierenden Daten erfolgt (III) die Analyse der Daten, die von rein deskriptiven Analysen bis

\footnotetext{
${ }^{3}$ http://prove-it.org/

${ }^{4}$ https://www.fzi.de/de/forschung/projekt-details/ran/

${ }^{5}$ https://www.fzi.de/de/forschung/projekt-details/bigpro-einsatz-von-big-data-technologien-zumstoerungsmanagement-in-der-produktion/

${ }^{6} \mathrm{https}: / / \mathrm{www} . f z i . d e / L S O$

${ }^{7} \mathrm{http} / / / \mathrm{www}$.proasense.eu/

${ }^{8}$ https://www.fzi.de/forschung/projekt-details/biggis/

${ }^{9} \mathrm{http} / / /$ www.reapp-projekt.de/index.php
} 


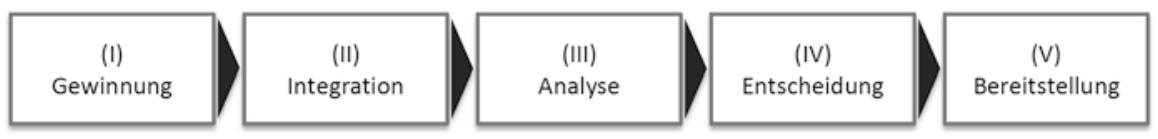

Abb. 15.1 Typische Datenverarbeitungsprozesse in Industrie 4.0 Anwendungen

hin zu komplexen Vorhersagen (prädiktive Analyse) reichen kann. Im vorletzten Schritt (IV) Entscheidungen erfolgt die präskriptive Analyse - entweder mithilfe einfacher regelbasierter Verfahren oder mithilfe fortgeschrittener Optimierungsverfahren. Die Phase (V) Bereitstellung steht am Ende des Entscheidungsprozesses und garantiert eine schnelle und bedarfsgerechte Kommunikation der notwendigen Informationen am Ort der Ausführung bzw. eine automatisierte Instanziierung der Entscheidung.

Der Aufwand für die Schritte Gewinnung und Integration hängt stark von der Anzahl der relevanten Datenquellen, dem Grad der Strukturierung der Daten sowie vom Umfang der für die nachfolgenden Schritte benötigten Daten ab. Je nach Anwendung kann zudem nach dem Schritt Analyse direkt die Bereitstellung folgen, z. B. in Form einer Visualisierung des Analyseergebnisses für menschliche Entscheider. Auch ist es möglich, dass eine automatisierte Weiterverarbeitung des Analyseergebnisses erfolgt. So kann eine Prognose unmittelbar als Eingangsgrößen in Steuerungs- oder Planungsverfahren dienen. Unabhängig vom Anwendungsfall sind bei einer Verknüpfung der Prozessschritte zu einer Datenverarbeitungskette jedoch insbesondere die folgenden Eigenschaften wünschenswert:

- Technische Adaptierbarkeit: Ein durchgängiges Datenverarbeitungssystem muss die Möglichkeit bieten, Konzepte und Werkzeuge einfach an sich ändernde technisch oder betriebliche Rahmenbedingungen anzupassen. Beim Störungsmanagement für Transportprozesse muss das System zum Beispiel mit sich wandelnden Netzwerken aus unterschiedlichen Logistikdienstleistern, Speditionen und deren teilweise häufig wechselnden Frachtführern umgehen können. In diesem Bereich unterscheiden sich nicht nur die IT-Voraussetzung zwischen großen Anbietern und Kleinbetrieben mit nur einer einzigen Zugmaschine im Fuhrpark. Teilweise unterscheiden sich auch die Geschäftsund Vertragsbedingungen sowie die betroffenen Logistikprozesse erheblich.

- Funktionale Adaptierbarkeit durch die Business User: Die zu entwickelnden Werkzeuge für die einzelnen Phasen müssen neben einer eingängigen Bedienbarkeit durch Endanwender die Möglichkeit der Berücksichtigung von Wissen und Domänenexpertise bieten. Zyklische, saisonale Trends (z. B. Absatzspitzen zu bestimmten Jahreszeiten) oder auch kurzfristig auftretende aber maschinell gut repräsentierte Ereignisse (z. B. Änderung der Verkehrslage) sind über automatisierte Modelle gut abbildbar. Doch gerade wichtiges Wissen über ,weiche“ Veränderungen - z. B. die Stimmungslage bei wichtigen Kunden - kann nur von Domänenexperten eingebracht werden. Die Möglichkeit der technischen Integration dieses Wissens ist daher nicht zuletzt aus Akzeptanzgründen beim späteren Anwender unabdingbar. 
- Generalisierbarkeit: Konzepte und Werkzeuge müssen auf unterschiedliche Fragestellungen innerhalb einer Domäne anwendbar sein. Im Anwendungsfeld Logistik zum Beispiel sollten einheitliche oder ineinander konsistent überführbare Datenmodelle für langfristige Analysen und Entscheidungen im Supply Chain Management und für sehr kurzfristigen Analysen und Entscheidungen im Störungsmanagement zur Verfügung stehen. Basismodelle und Infrastruktur sollten auch über Domänengrenzen hinweg wiederverwendbar sein.

- Robustheit: Die zu entwickelnden Systeme müssen robust gegenüber Schwankungen in der Qualität der gelieferten Daten und Zwischenergebnisse sein. Die Qualität einer geschätzten Ankunftszeit in einem Transportprozess hängt zum Beispiel sehr stark von der Qualität der Fahrzeitprognosen und damit von der Qualität aktueller Verkehrsinformationen ab. Das sollte in den nachfolgenden Verarbeitungsprozessen bekannt sein und so Berücksichtigung finden können.

- Echtzeitfähigkeit: Im Kontext Industrie 4.0 besteht die Anforderung einer Nah-Echtzeitfähigkeit in der Regel für alle Komponenten oder Module eines Datenverarbeitungssystems. Eine besondere Herausforderung stellt die Echtzeitfähigkeit für modularisierbare Verfahren in den Bereichen Analyse und Optimierung dar, denn in der Regel hängt die Leistungsfähigkeit von Verfahren hinsichtlich Laufzeit und Qualität von der konkreten Ausprägung von Problemstellungen ab. Hier kann die semantische Beschreibung der Problemstellung helfen, automatisiert passende Verfahren in Abhängigkeit der Problemausprägung und der konkreten Probleminstanz auszuwählen (siehe Abschn. 15.2.2).

Auf der Ebene der einzelnen Datenverarbeitungsschritte existieren aktuell sowohl forschungs- als auch praxisseitig vielversprechende Ansätze, die diese Aspekte oder bestimmte Ausschnitte erfolgreich adressieren. Eine große Herausforderung, und gleichzeitig ein großes Potenzial, besteht jedoch in der Zusammenführung der Einzelschritte in eine ganzheitliche datengetriebenen System- und Toolinfrastruktur.

\subsubsection{Decision Support Pipeline - Manifestation einer durchgängigen Datenverarbeitungskette}

In der Decision Support Pipeline manifestiert sich unsere Vision einer datengetriebenen System- und Werkzeuginfrastruktur, die die zuvor beschriebenen Eigenschaften mitbringt und den Anforderungen gerecht wird. Im Folgenden geben wir zunächst einen Überblick über die wichtigsten Komponenten und beschreiben danach deren konzeptionelle und funktionale Basis.

\subsubsection{1 Übersicht}

Abb. 15.2 zeigt die Hauptkomponenten der Decision Support Pipeline. Die weiß hinterlegten Module lassen einen engen Zusammenhang zu den zuvor identifizierten typischen Prozessschritten erkennen: Ein Modul adressiert gemeinsam die Gewinnung und Integration der Daten, ein zweites die Analyse, ein weiteres das Thema Entscheidungsunterstützung 


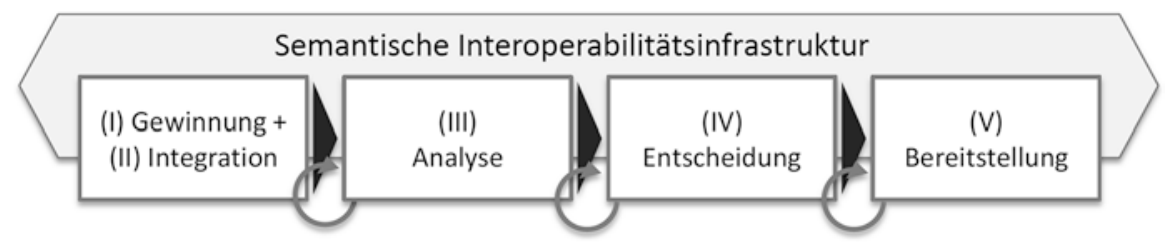

Abb. 15.2 Hauptkomponenten und Ablauf einer Decision Support Pipeline

und ein letztes adressiert die Bereitstellung der Ergebnisse. Der Zuschnitt der Module ergibt sich aus der engen Verwandtschaft der verwendeten Methoden.

Die in der Abbildung grau hinterlegte semantische Interoperabilitätsinfrastruktur stellt das verbindende Element über die Module hinweg dar: Die Interoperabilitätsinfrastruktur baut auf Sprachen und Technologies des semantischen Web Stack ${ }^{10}$ (W3C 2007) auf, die im Rahmen der W3C Data Activity ${ }^{11}$ entworfen und standardisiert wurden. Sie nutzt das Resource Description Framework (RDF) (Klyne 2004; Lassila \& Swick 2002) und Ontologiesprachen wie die Web Ontology Language (OWL) (Owl 2 web ontology language document overview 2009; Dean \& Schreiber 2004; W3C OWL Working Group 2012) als Datenbeschreibungs- und Austauschformat. Dies ermöglicht die Erstellung eines übergreifenden Meta-Modells, welches neben den Eingangs- und Ausgangsdaten auch die Modelle und Werkzeuge auf Modulebene sowie die (Zwischen-)Ergebnisse des Analyseund Entscheidungsmoduls in einem einheitlichen Format beschreibt. So wird einerseits die angestrebte „Durchlässigkeit“ des Datenverarbeitungsprozesses gewährleistet, andererseits erlaubt dieser Modellansatz eine intensive Unterstützung des Anwenders bei der Konfiguration von Methoden oder Diensten. So können bei der Komposition von Analyseund Entscheidungsmodellen nicht nur Konsistenzprüfungen innerhalb des Moduls vorgenommen werden, sondern es kann auch geprüft werden, ob die für die gewählten Werkzeuge benötigten Daten im richtigen Format und in ausreichender Qualität vorliegen. Sollen beispielsweise stochastische Optimierungsverfahren im Entscheidungsmodul zum Einsatz kommen, so müssen im Analysemodul entsprechende statistische Verteilungen aus den Daten hergeleitet werden.

Die in der Abbildung dargestellten Feedbackschleifen zwischen den Modulen sollen verdeutlichen, dass die Decision Support Pipeline nicht zwangsläufig linear und unidirektional verläuft. Zum Beispiel hängt die Qualität der Lösung und die Laufzeit bei vielen Optimierungsverfahren von der zeitlichen Auflösung und dem Aggregationsniveau der Eingangsdaten ab. Dementsprechend gibt es Anwendungen, in denen es sinnvoll ist, dass der Anwender in einem iterativen Prozess auch bei der Auswahl der für das Entscheidungsmodells passenden Granularität der Inputdaten unterstützt wird.

\footnotetext{
${ }^{10} \mathrm{http}: / / \mathrm{www} . \mathrm{w} 3$. org/standards/semanticweb/

${ }^{11} \mathrm{http}: / /$ www.w3.org/2013/data/
} 


\subsubsection{Funktionale Beschreibung der Komponenten}

Aufbauend auf den in Abb. 15.2 dargestellten Schritten beschreibt dieses Kapitel die wesentlichen Aufgaben der einzelnen Decision Support Pipeline Komponenten.

\section{Gewinnung und Integration:}

Ein wesentlicher Bestandteil dieser Prozessschritte ist die Entwicklung einer applikationsunabhängigen und skalierbaren Datenakquiseinfrastruktur zur Erfassung und Aggregation von verteilten heterogenen Datenströmen. Diese Datenströme werden von unterschiedlichen Datenquellen erfasst und in weiteren Schritten ,veredelt“. Grundlage hierfür bildet die Definition eines einheitlichen Ontologie-basierten semantischen Datenstrukturmodels, auf dessen Basis die Akquise, Aggregation, Repräsentation, Verfeinerung und Verteilung von gewonnenen Daten vollzogen werden kann. Ein solches semantisches Datenstrukturmodell bildet die Basis für eine Reihe von wesentlichen Funktionen:

(i) Es ermöglicht die Transformation von Rohdatenströmen in sinnvolle und aussagekräftige semantische Instanzdaten, sogenannte semantische Graphenstrukturmodelle (vgl. RDF (Klyne 2004; W3C 2014)), die mithilfe von gängigen industriespezifischen Klassifikationssystemen und Vokabularen annotiert werden.

(ii) Es ermöglicht die Entkopplung von Akquise-, Aggregations- und Verarbeitungslogik durch den Einsatz des Konzepts der Data Uniformity, da Daten und Datensemantik von der eigentlichen Verarbeitungslogik getrennt werden. Datenmodelle enthalten neben den reinen Daten (sog. Observables) auch Informationen zu ihrer intentionalen Semantik.

(iii) Die Kopplung mit axiomatisch definierten Wissensbasen (vgl. Baader et al. 2003; Krötzsch et al. 2014; Rudolph 2011) erlaubt die Aufstellung und Überprüfung von Konsistenzanforderungen an gewonnenen und aggregierten Daten durch Inferenzmaschinen, sogenannte DL Reasoner. ${ }^{12}$ Ferner lässt sich die durch Ontologien beschriebene formale Semantik nutzen, um implizit enthaltenes Wissen in gewonnen Daten sichtbar zu machen (siehe Zander \& Awad 2015).

(iv) Es ermöglicht die Miteinbeziehung von Qualitätsmerkmalen (sog. Data Quality Indicators) auf Basis derer eine Abschätzung hinsichtlich der Verlässlichkeit und Vertrauenswürdigkeit gewonnener Daten möglich ist. Dies ist zum Beispiel für die frühzeitigen Erkennung von anstehenden Wartungsarbeiten bei Anlagen und Maschinen (Predictive Maintenance) hilfreich, da durch eine präzisere und quantitativ wie qualitativ höherwertige Datenbasis eine bessere maschinelle Datenanalyse sowie Planung gewährleistet werden kann.

\section{Analyse:}

Aufbauend auf der Datenakquiseinfrastruktur unterstützt eine Fabrik von Modellen und Methoden zur Visualisierung und (semi-)automatisierten Analyse der Daten deren

\footnotetext{
${ }^{12}$ Beispiele zur Überprüfung von Konsistenzanforderungen an aggregierten Daten mittels ontologischer Semantik und Inferenz finden sich in (Zander \& Awad 2015 und Zander et al. 2015).
} 
Verdichtung sowie deskriptive und prädiktive Analysen. Der Methodenkatalog umfasst dabei auch zu Analyseprozessen orchestrierte Modelle und Methoden (sog. ,serviceoriented analytical architecture") um flexibel konfigurierbar analytische Fragestellungen im Bereich Industrie 4.0 zu adressieren. Zentrales Element der Fabrik (neben dem Katalog mit analytischen/orchestrierten Techniken) ist ein „Smart Model Selector“. Dieser soll mit den vorhandenen Daten entweder autonom die integrierte Modell- und Datenauswahl vornehmen oder die Auswahl und Konfiguration weitgehend unterstützen und auch ,beste Alternativen“" inklusive erklärenden Texte und Statistiken generieren.

Neben der Abstimmung mit den Datenmodellen in der Datenakquiseinfrastruktur (z. B. zur Gewichtung von Datenquellen basierend auf deren Qualitätsmerkmalen) erfolgt eine Koordination auch mit den zu lösenden Optimierungsproblemen und -modellen. Dieses Vorgehen bei der Auswahl und Konfiguration von analytischen Modellen ist grundlegend neu, da hierbei eine problemsensitive Daten- und Modellauswahl erfolgt. Hierdurch soll die Daten- und Analysemodellauswahl zielgerichteter auf die Optimierungsmodelle abgestimmt werden und letztlich komplexe Planungs- und Entscheidungsprobleme im Kontext von Industrie 4.0 besser gelöst und Prozesse effizienter und robuster durchführbar werden.

Beispielsweise ist bei heutigen Planungs- und Steuerungsaufgaben in der Logistik nicht klar, welcher Planungshorizont bei der Bestimmung von Produktlosgrößen auf Produktionsanlagen bei unsicherer Nachfrage und weiteren stochastischen betrieblichen Kennzahlen gewählt werden soll, um nahezu optimale und robuste Produktionsprogrammplanungen zu bestimmen. Dies hängt neben Charakteristika des Optimierungsproblems (z. B. Rüstkosten und -zeiten) maßgeblich auch von dem Prognosehorizont der Kennzahlen ab, bzw. wie sich die Unsicherheit der Prognosen mit steigendem Prädiktionshorizont (LeadTime) verhält. Umgekehrt sollten statistische Prognosemodelle wiederum für bestimmte Horizonte, Robustheitsanforderungen und Prognosegütekriterien konfiguriert werden, um eine hohe Prognosegüte erreichen zu können. Damit können letztlich nur abgestimmte, integrierte Fahren der Prognose und mathematischer Programmierung/Entscheidung zu bestmöglichen Lösungen führen.

\section{Entscheidung/Optimierung:}

Die Vielzahl an integrierten und transformierten Daten schafft die Basis zum intelligenten Einsatz automatisierter Planungs- und Optimierungsverfahren. Allerdings steigt durch die Heterogenität der Systeme, immer kürzer werdende Entwicklungszyklen und wechselnde Anforderungen der Anspruch an die Flexibilität und Erweiterbarkeit dieser Verfahren.

Viele aktuelle Forschungs- und Praxisansätze zielen bereits auf eine einfache Adaptierbarkeit von Optimierungs- und Entscheidungsmodelle in sehr unterschiedlichen Anwendungskontexten ab (siehe z. B. (Heckmann 2015; Amberg 2015; Meyer 2015; Irnich 2008)). Für eine korrekte Konfiguration der Optimierungsmodelle, sowie die Anbindung an vor- und nachgelagerte Prozesse und die Auswahl eines passenden Lösungsverfahrens ist jedoch i. d. R. die Expertise von Optimierungs- und Datenspezialisten notwendig. Selbst kleinere Anpassungen können häufig nicht von Domänenexperten vorgenommen 
werden. Kernvision im Bereich Optimierung ist demgemäß die weitgehende Unterstützung des Fachanwenders bei der Konfiguration des Entscheidungsschrittes durch

(i) die semantische Beschreibung von wiederkehrenden Modellbausteinen, um diese im jeweiligen Anwendungskontext (z. B. Störungsmanagement in Transportnetzwerken) automatisch zu vollständigen Optimierungsmodellen zu kombinieren und eine Prüfung des Modelles auf Konsistenz und eine Prüfung der Verfügbarkeit der Daten aus den vorgelagerten Schritten vornehmen zu können, sowie

(ii) die automatisierte Auswahl aus einer Menge an Lösungsverfahren (exakte Verfahren, Heuristiken, etc.) auf Basis von geeigneten Leistungskennzahlen (siehe z. B. Dunke und Nickel 2015) und erwarteten Antwortzeiten für unternehmensrelevante Instanzen.

\section{Bereitstellung:}

Die unbedingte Notwendigkeit der bedarfsgerechten Informationsbereitstellung wird auch im Beitrag „Erweiterte Horizonte - Ein technischer Blick in die Zukunft der Arbeit“ von Maettig et al. adressiert. In der Decision Support Pipeline kann die Funktion des letzten Moduls im Wesentlichen zwei Ausprägungen annehmen: Entweder werden die Ergebnisse aus Analyse- oder Entscheidungsmodul dem Anwender zur manuellen Weiterverarbeitung visuell aufbereitet präsentiert oder die Ergebnisse werden in digitaler Form an andere Systeme (z. B. ERP-Systeme, Maschinen, etc.) zurückgespielt. Ersteres wird hauptsächlich im Modul Analyse adressiert. Letzteres ist eng verwandt mit den Methoden aus dem Modul Gewinnung und Integration, denn hier müssen die Analyse- oder Optimierungsergebnisse zurück in die ursprünglichen, von den außenstehenden Systemen verarbeitbaren, Formate transformiert werden.

\section{Semantische Annotation und ontologie-basierte Modellbildung:}

Eine der größten Herausforderungen des vorgestellten Ansatzes ist die Sicherstellung der Durchgängigkeit der involvierten Modelle und Werkzeuge in der Interoperabilitätsinfrastruktur. Um die Durchgängigkeit sowie die Anpassbarkeit an unterschiedliche Systemkontexte und Problemstellungen zu gewährleisten, werden die eingangs erwähnten semantischen Beschreibungssprachen und Technologien verwendet. Dieses Vorgehen vereinfacht einerseits darauf aufbauende Visualisierungsmöglichkeiten als auch die Datenhaltung von prozessierten Daten, da neben den reinen Daten auch deren Bedeutung in einer formalen, maschinenverarbeitbaren Form gespeichert ist (Baader et al. 2003; Domingue et al. 2011; Hitzler et al. 2010; Krötzsch et al. 2014).

Hinsichtlich der Anforderung an die Echtzeitfähigkeit der Analyse- und Entscheidungskomponenten erlaubt die semantische Beschreibung des Problems und der Daten, weitgehend automatisiert passende Verfahren für eine konkrete Probleminstanz auszuwählen und $\mathrm{zu}$ integrieren. 


\subsection{Anwendungsszenarien}

Im vorangegangenen Abschnitt beschreiben wir - basierend auf unseren Erfahrungen über Projekte aus unterschiedlichen Anwendungsfeldern hinweg - die Vision und mögliche Umsetzung einer durchgängigen Decision Support Pipeline in abstrakter Form. Ziel dieses Abschnittes ist es anhand dreier Anwendungsszenarien aufzuzeigen, wie die Decision Support Pipeline die Entscheidungsunterstützung von morgen in Logistik und Produktion konkret positiv verändern wird.

\subsubsection{Supergrid Logistics}

Laut der aktuellen Ausgabe des DHL Logistik Trendradars werden Logistics Supergrids eine neue Generation von Logistikdienstleistern hervorbringen, deren Fokus auf der Orchestrierung weltweit verteilter Supply Chains und Dienstleister liegt (Bubner et al. 2014). Basis von Logistics Supergrids ist ein gut strukturiertes und modular konfigurierbares Logistik-Service-Portfolio, aus dem durch die Auswahl von Services „on demand“ kundenspezifische Netzwerke innerhalb des Supergrids entstehen (Logistics-as-a-Service). Kernaufgabe des Supergrid Anbieters ist die effektive und effiziente Planung und Steuerung dieser hochkomplexen, sich wandelnden und weltweit verteilten Netzwerke eine Aufgabe, die von der strategischen Designentscheidungen unter Berücksichtigung von Risiken bis hin zum operativen Störungsmanagement reicht.

Für das Störungsmanagement in Transportketten bieten Technologien aus dem Kontext Industrie 4.0 die Basis, um Daten über den Zustand der weltweit verteilten Logistiksysteme und der transportierten Güter in Nahe-Echtzeit zu sammeln. Entscheidend für die erfolgreiche Steuerung dieser Netzwerke ist es jedoch, diese Daten zu guten - datengetriebenen - Entscheidungen zu veredeln: Dafür müssen die Ausführungsdaten über die Netzwerkpartner hinweg gesammelt und mit den Plandaten aus den Systemen der Partner integriert werden. Dabei müssen die gewonnenen Informationen hinsichtlich ihrer Kritikalität für das System analysiert und für ein IT-gestütztes Störungsmanagement aufbereitet werden. Die Reaktionsmaßnahmen müssen an die Orte der Ausführung verteilt werden. Das Konzept der möglichst freien Konfigurierbarkeit der Logistics Services stellt dabei höchste Anforderungen an die Systeme zur Entscheidungsunterstützung.

Ein Disponent, der für das Adhoc-Netzwerk eines Kunden innerhalb des Logistics Supergrid zuständig ist, nutzt die Decision Support Pipeline, um eine kundenspezifisches Störungsmanagement zu installieren: Über das Metamodell definiert er, woher die Planund Ausführungsdaten stammen, welche Analyseschritte zur Erkennung von Abweichungen nötig sind und welche Abweichungen als kritisch zu betrachten sind. Die kritische Abweichungen werden an die Störungsbehebung (Modul: Entscheiden/Optimieren) übergeben. Innerhalb dieses Moduls legt der Anwender fest, welche Handlungsalternativen im Störungsfall Anwendung finden soll, d. h. er definiert den Lösungsraum und die Kriterien nach denen Lösungen ausgewählt werden (Zielfunktionsdefinition). Sowohl im Modul 
Analyse als auch im Modul Entscheidung wird der Anwender dabei unterstützt, zu prüfen, ob die für die Modelle und Methoden relevanten Daten aus den Vorschritten verfügbar sind und insbesondere ob die Daten in ausreichender Qualität und in der richtigen Form zur Verfügung stehen. Außerdem werden die für das kundenspezifische Netzwerk passenden Lösungsverfahren automatisch ausgewählt.

\subsubsection{Entscheidungsunterstützung bei der Produktionsplanung}

Wie im Beitrag „Der Mensch in Interaktion mit autonomen Planungs- und Steuerungssystemen für Cyber-Physische Produktionssysteme“ von Susanne Vernim, Christiane Dollinger, Andreas Hees und Günther Reinhart diskutiert, sehen sich Produktionsmitarbeiter wie bspw. Meister und Produktionsplaner in Industrie 4.0 getriebenen Planungs- und Fertigungsverfahren komplexen Aufgaben gegenüber, die aus der zunehmenden Produktvielfalt und dem Wunsch vieler Kunden nach Produktindividualität resultieren. Diese neuen bzw. veränderten Produktionskonzepte stellen gezielte Anforderungen an Aspekte wie Flexibilität und Autonomie und erfordern das Treffen schneller Entscheidungen sowie eine permanente Fortschrittskontrolle. Für den Produktionsplaner sind hierfür Daten zu filtern, aufzubereiten und zu repräsentieren, sodass dieser unmittelbaren Zugriff auf alle relevanten Daten erhalt und damit jederzeit die aktuelle Situation erfassen und nachvollziehen kann - im Idealfall unter Berücksichtigung persönlicher Präferenzen der NutzerInnen und deren Arbeitskontexten. ${ }^{13}$

Wie die Autoren aufzeigen, treten an die Stelle der operativen Ausführung von Planungs- und Steuerungsaufgaben vermehrt Kontroll- und Überwachungsaufgaben, die insbesondere eine ganzheitliche Prozess- und Modellsicht berücksichtigen müssen. Die Definition eines übergeordneten semantischen Meta-Modells erlaubt die Erstellung eines solch ganzheitlichen Prozessmodells, welches die einzelnen Phasen (siehe Abb. 15.2) abstrahiert und miteinander verbindet. Durch das Konzept der Data Uniformity (siehe Abschn. 15.2.2.2) kann eine einheitliche Abfragesprache, beispielsweise auf Basis der Standard-RDF-Abfragesprache SPARQL (Prud'hommeaux und Seaborne 2008), für alle Module oder Phasen genutzt werden.

Weiter führen die Autoren an, dass für das Erkennen von unerwartet auftretenden Situationen und deren Behandlung bzw. die Reaktion darauf ein grundlegendes Verständnis von informations-technischen Zusammenhängen und der Funktionsweise von IT-Systemen und deren Arbeitsschritten unerlässlich ist. Eine durchgängige Datenverarbeitungskette hilft, Datenverarbeitungsprozesse nachvollziehbarer und besser kontrollierbar zu machen. Das in Ontologien enthaltene geteilte Verständnis über die relevanten Entitäten einer Domäne sowie deren Eigenschaften manifestiert ein solches grundlegendes Verständnis. Die Flexibilität und Ausdrucksmächtigkeit von Ontologiesprachen und den zugrunde

\footnotetext{
${ }^{13}$ Vgl. auch den Beitrag von Maetting et al.
} 
liegenden Beschreibungsrahmenwerken erlaubt es, Planungs- oder Fertigungsprozesse aus unterschiedlichen Perspektiven und in Granularitäten zu beschreiben.

\subsubsection{Predictive Maintenance}

Predictive Maintenance zielt auf die Prognose der Ermüdung und Disfunktionalität von Bauteilen. Durch rechtzeitige bzw. vorzeitige Wartung und Reparatur bzw. die proaktive Unterstützung des Austauschs von Komponenten können Ausschuss in der Produktion verringert, Service-Levels mit Kunden besser eingehalten sowie Kosten für Service und Instandhaltung verringert werden.

Hierfür müssen sowohl Zustandsmodelle von Maschinen und Prozessen als auch historische und aktuelle Zustandsdaten der Maschinen vorliegen. Unter Verwendung geeigneter Algorithmik aus dem statistischen Lernen können diese Massendaten wertvolle Informationen bzgl. einer Einschätzung der Entwicklung der Komponenten liefern. Werden mathematischen Entscheidungsmodellen berücksichtigt, die Aussagen darüber treffen, wie und wann bestimmte Bauteile aus unterschiedlichen Gesichtspunkten (Kösten, Sicherheit, Planbarkeit etc.) ausgewechselt werden sollten bzw. könnten, können Wartungsprozesse inklusive der unterstützenden Prozesse wie die Produktion und Lagerungen von Ersatzteilen besser abgestimmt und effizienter durchgeführt werden.

So verzeichnen befragte Unternehmen schon heute knapp $30 \%$ weniger Kosten für Service und Instandhaltung durch Predictive Maintenance und beispielweise eine um knapp $20 \%$ höhere Einhaltung von Produktionsplänen, wenn Liefertermine für Kunden mit Materialverfügbarkeits- und Echtzeit-Herstellungsbedingungen integriert werden (siehe SAP 2013). Aktuell wird allerdings erst damit begonnen, das Potenzial von Predictive Maintenance zu erschließen. Hierbei sind integrierte Modelle und eine durchgängige Decision Support Pipeline als der Schlüssel zum Erfolg anzusehen.

\subsection{Zusammenfassung}

Eine Schlüsseltechnologie im Kontext Industrie 4.0 ist die Transformation großer Datenmengen zu guten unternehmerische Entscheidungen. Um dies in dynamischen Geschäftsumfeldern gewährleisten zu können, werden höchste Anforderungen an die Datenverarbeitungsprozesse gestellt: Neben der einfachen technischen und funktionalen Adaptierbarkeit und der Echtzeitfähigkeit des Systems sind Robustheit gegenüber Schwankungen in der Qualität der Eingangsdaten und eine Generalisierbarkeit der Konzepte und Werkzeuge anzustreben. Um diese Anforderungen zu adressieren, berücksichtigt das Konzept der Decision Support Pipeline alle Datenverarbeitungsschritte und verbindet diese über ein ganzheitliches Meta-Modell. Diese Modell ermöglicht es, (1) die Daten und ihre Semantik von den konkreten Methoden aus den Modulen Analyse und Entscheidung zu entkoppeln und (2) den Anwender bei der Komposition von Modellen und Verfahren in den Modulen Analyse und Entscheidung zu unterstützen. 
Den Mehrwert einer durchgängigen Datenverarbeitungsinfrastruktur haben wir anhand von drei Anwendungsszenarien aufgezeigt: Im ersten Anwendungsszenario bietet die einfache Adaptierbarkeit der Decision Support Pipeline die Basis für das Angebot von komplexen Echtzeitservices in der Logistik. Im zweiten Szenario liegt der Nutzen in der bedarfsgerechten Aufbereitung und Bereitstellung von Produktionsdaten, um einem menschlichen Planer den Zustand der Produktion transparent zu machen, während im letzten Beispiel ein besonderer Nutzen dadurch entsteht, dass der Anwender bei der Komposition von abgestimmten Analyse- und Entscheidungsmodellen unterstützt wird.

\section{Literatur}

Amberg, B. (2015). Ressourceneinsatzplanung im öffentlichen Personennahverkehr: Ähnlichkeitsaspekte in der fahrplanübergreifenden Optimierung. PhD thesis, Paderborn, Universität Paderborn, Diss., 2014.

Baader, F., Calvanese, D., McGuinness, D., Nardi, D., \& Patel-Schneider, P. (2003). The description logic handbook: Theory, implementation and applications. Cambridge: Cambridge University Press.

Beste, D. (2014). Lässt sich die Datenflut der Industrie 4.0 bändigen? Technical report, Springer für Professionals - Schwerpunkt Maschinenbau.

Bubner, N., Helbig, R., \& Jeske, M. (2014). LOGISTICS TREND RADAR. Delivering insight today. Creating value tomorrow! Version 2014. Technical report, DHL Customer Solutions \& Innovation.

Dean, M., \& Schreiber, G. (2004). Owl web ontology language reference. W3c recommendation, Web Ontology Working Group, World Wide Web Consortium.

Domingue, J., Fensel, D., \& Hendler, J. A. (Hrsg.) (2011). Handbook of semantic web technologies. Berlin: Springer.

Dunke, F., \& Nickel, S. (2015). A general modeling approach to online optimization with lookahead. Omega.

European Commission. (2013) Report from the Workshop on Cyber-Physical Systems: Uplifting Europe's Innovation Capacity. Communications Networks, Content \& Technology DirectorateGeneral, Unit A3-DG CONNECT, December 2013.

Heckmann, I. (2015). Towards Supply Chain Risk Analytics - Fundamentals, Simulation, Optimization. $\mathrm{PhD}$ thesis, Karlsruher Institut für Technologie. To be published.

Hitzler, P., Krötzsch, M., \& Rudolph, S. (2010). Foundations of semantic web technologies. Boca Raton: CRC Press.

Irnich, S. (2008). A unified modeling and solution framework for vehicle routing and local search based metaheuristics. INFORMS Journal on Computing, 20(2), 270-287.

Klyne, J. J. C. G. (2004) Resource description framework (rdf): Concepts and abstract syntax. Technical report, W3C.

Krötzsch, M., Simancik, F., \& Horrocks, I. (2014). Description logics. IEEE Intelligent Systems, $29,12-19$.

Laney, D. Vortrag: Information economics, big data and the art of the possible with analytics. https:// www-950.ibm.com/events/wwe/grp/grp037.nsf/vLookupPDFs/Gartner_Doug-\%20Analytics/ \$file/Gartner_Doug-\%20Analytics.pdf. Zugegriffen: 11. Dez. 2015).

Lassila, O., \& Swick, R. R. (2002). Resource Description Framework (RDF) model and syntax specification. http://www.w3.org/TR/REC-rdf-syntax/. Zugegriffen: 13. Dez. 2015. 
Meyer, M. (2015). Milk run design: Definitions, concepts and solution approaches. PhD thesis, Karlsruher Institut für Technologie, 2015.

P. A. C. (PAC). (2013). It innovation readiness index. Technical report, Pierre Audoin Consultants (PAC).

Prud'hommeaux, E., \& Seaborne, A. (2008) SPARQL Query Language for RDF. W3C Recommendation.

Rudolph, S. (2011). Foundations of description logics. In A. Polleres, C. d'Amato, M. Arenas, S. Handschuh, P. Kroner, S. Ossowski, \& P. F. Patel-Schneider (Hrsg.), Reasoning web. Semantic technologies for the web of data - 7th international summer school 2011 (Bd. 6848 of LNCS, S. 76-136). Berlin: Springer.

SAP R \& D, Manufacturing, and Service. (2013) Idea to performance - maximizing opportunity in a new, technology-driven industrial revolution. Technical report, SAP.

Schreier, J. (2013). Industrie 4.0 - Ist die Datenflut überhaupt noch zu bewältigen? Technical report, MM MaschinenMarkt - IT-Agenda Fertigung.

W3C. (2007). Semantic web stack.

W3C. (2012). OWL Working Group. OWL 2 Web Ontology Language Document Overview (2. Aufl.) - W3C Recommendation 11 Dec. 2012.

W3C. (2014). RDF 1.1 Concepts and Abstract Syntax. https://www.w3.org/TR/rdf11-concepts/. Zugegriffen: 21. Okt. 2017

Zander, S., Heppner, G., Neugschwandtner, G., Awad, R., Essinger, M., \& Ahmed, N. (2015). A model-driven engineering approach for ros using ontological semantics. In Proceedings of the 6th International Workshop on Domain-Specific Languages and models for ROBotic systems (DSLRob-15) co-located with the IEEE/RSJ International Conference on Intelligent Robots and Systems (IROS), 2015.

Zander, S., \& Awad, R. (2015). Expressing and reasoning on features of robot-centric workplaces using ontological semantics. In IEEE/RSJ International Conference on Intelligent Robots and Systems, Hamburg, Germany, 2015.

Open Access Dieses Kapitel wird unter der Creative Commons Namensnennung 4.0 International Lizenz (http://creativecommons.org/licenses/by/4.0/deed.de) veröffentlicht, welche die Nutzung, Vervielfältigung, Bearbeitung, Verbreitung und Wiedergabe in jeglichem Medium und Format erlaubt, sofern Sie den/die ursprünglichen Autor(en) und die Quelle ordnungsge-mäß nennen, einen Link zur Creative Commons Lizenz beifügen und angeben, ob Änderungen vorgenommen wurden.

Die in diesem Kapitel enthaltenen Bilder und sonstiges Drittmaterial unterliegen ebenfalls der genannten Creative Commons Lizenz, sofern sich aus der Abbildungslegende nichts anderes ergibt. Sofern das betreffende Material nicht unter der genannten Creative Commons Lizenz steht und die betreffende Handlung nicht nach gesetzlichen Vorschriften erlaubt ist, ist für die oben aufgeführten Weiterverwendungen des Materials die Einwilligung des jeweiligen Recht-einhabers einzuholen.

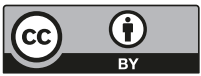

